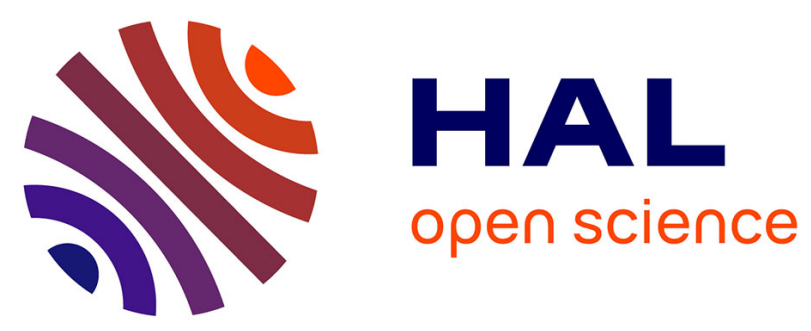

\title{
An experimental study of secondary oil migration in a three-dimensional tilted porous medium
}

\author{
Jianzhao Yan, Luo Xiaorong, Weimin Wang, Renaud Toussaint, Jean \\ Schmittbuhl, Guy Vasseur, Fang Chen, Alan Yu, Likuan Zhang
}

\section{- To cite this version:}

Jianzhao Yan, Luo Xiaorong, Weimin Wang, Renaud Toussaint, Jean Schmittbuhl, et al.. An experimental study of secondary oil migration in a three-dimensional tilted porous medium. AAPG Bulletin, 2012, 96 (5), pp.773-788. 10.1306/09091110140 . hal-00701961

\section{HAL Id: hal-00701961 \\ https://hal.science/hal-00701961}

Submitted on 30 Nov 2020

HAL is a multi-disciplinary open access archive for the deposit and dissemination of scientific research documents, whether they are published or not. The documents may come from teaching and research institutions in France or abroad, or from public or private research centers.
L'archive ouverte pluridisciplinaire HAL, est destinée au dépôt et à la diffusion de documents scientifiques de niveau recherche, publiés ou non, émanant des établissements d'enseignement et de recherche français ou étrangers, des laboratoires publics ou privés. 


\section{An Experimental Study of Oil Secondary Migration in a Three Dimensional Tilted Porous Medium}

Jianzhao Yan, Xiaorong Luo, Weimin Wang, Renaud Toussaint, Jean Schmittbuhl, Guy Vasseur, Fang Chen, Alan Yu, Likuan Zhang

Abstract: A three-dimensional physical experiment was carried out to study the oil secondary migration under an impermeable inclined cap. Light colored oil was released continuously at a slow rate of about $0.1 \mathrm{ml} / \mathrm{min}$ from a point at the basis of initially water saturated porous model. With the buoyancy as a primary driven force, a vertical cylindrical shape of oil migration pathway was observed first and then a layer-shaped of lateral migration pathway beneath the top inclined sealing plate once the oil cluster had reached the top cap. The Magnetic Resonance Imaging (MRI) was used to observe the migration processes - e.g. morphology of the migration pathway, intermittency of oil bubbles and variation of oil saturation within the migration paths. Results show that the snap-off phenomenon (related to fast local imbibition processes) occurred more often during vertical migration than it is during lateral. The lateral migration pathway which parallels to the top inclined cap has a typical vertical thickness of $2-4 \mathrm{~cm}$ (i.e. roughly $40-80$ pores). This thickness is consistent with the prediction derived from the scaling laws related to the pore size and the Bond number. Along the lateral migration direction, the sectional area and the horizontal width of the migration pathway fluctuate drastically while the average oil saturation along the pathway remains almost the same. After stopping initial oil injection, the sectional area of migration pathway shrinks significantly. Therefore, it is 
believed that this drastic shrinking of the migration pathway is the main reason why only relative small volume of oil and gas has been lost during the secondary migration.

Keywords: Secondary migration, Analog model, MRI, Oil saturation, Instability biphasic flow

\section{Introduction}

Secondary migration is the movement of hydrocarbons as a separate fluid through water-saturated rocks, faults, or fractures. The lateral secondary migration in a specific carrier bed is considered to be the major process accounting for large-scale oil and gas accumulations in most reservoirs (Schowalter, 1979; Thomas and Clouse, 1995). However, the secondary migration typically occurs over a long geological time period and is impossible to be observed directly in situ. In the field, it is very difficult also to locate or identify any active migration pathway even the remaining structure left after the migration. Therefore, laboratory experiment gives a unique mean of observing real oil and gas migration processes in porous media and studying the transport mechanism (Schowalter, 1979; Dembicki and Anderson, 1989; Catalan et al., 1992).

As commonly expected, hydrocarbon lateral migration actually takes place in three-dimensional (3D) within strata, bounded by sealing rocks (Schowalter, 1979; England, 1987; Hindle, 1997; Carruthers and Ringrose, 1998). Due to the limitations of experimental model building and observation technique capability, it is difficult to design a proper $3 \mathrm{D}$ model to simulate secondary migration process fully appropriately. 
Most published experiments dealing with lateral migration were based on one-dimensional (1D) or two-dimensional (2D) models (Emmons, 1921; Lenormand, 1988; Catalan et al., 1992; Wagner et al., 1995, 1997; Thomas and Clouse, 1995; Meakin et al., 2000; Tokunaga et al. 2000; Zhang et al., 2003; Hou et al., 2004, 2005; Luo et al., 2004), which may not reflect the complexity of hydrocarbon lateral migration within the carrier-seal system. Therefore the main objective in this study is to carry out a 3D physical experiment using a relative large box model which should be closer to realistic than the previous experiments to investigate the characteristics of oil migration in the $3 \mathrm{D}$ space.

There were a few physical experiments of 3D immiscible flow which had been done and published by others under very different experimental conditions (system size) and with limited observational device capability. Frette et al. (1992) carried out an experiment in a glass box to study the migration process of non-wetting phase driven by buoyancy. The glass box was filled with cylindrical grains of Röhm Plexiglas Formmasse 7N Glasklar [poly(methylmethacrylate)] (PMMA) and saturated with dibutyl-phthalate as the non-wetting phase. The model was transparent because PMMA and dibutyl-phthalate have the same refraction index allowing the internal structure of the migration pathway to be observed directly. Stöhr et al. (2003) used the planar laser-induced fluorescence (PLIF) technique to simultaneously visualize the 3D pore-scale flow of two immiscible liquid phases in porous media. Although the PLIF technique is capable to reconstruct the $3 \mathrm{D}$ fluid flow pathway and can observe the internal structure and morphology directly by selecting the solid and liquid phases 
with the same refractive index, there are some strict requirements for the nature of the porous medium and the fluid, which were not satisfactorily met by this technique. The X-ray absorption technique (Selle et al., 1993) and Ultrasonic technique (Thomas and Clouse, 1995) were also tentatively used to observe the migration pathway structure in a porous model and to measure oil saturation, but only for the quasi-2D media. Sharma et al. (1997) and Hou et al. (2009) used an X-ray CT (Computed Tomography) technique to observe fluid flow and measure fluid saturation in the 3D porous medium. Although this CT technique has a high resolution, it is unsuitable to be applied for a large migration model since the Field Of View (FOV) is limited (Seright et al., 2002; Turner et al., 2004).

Comparing all the techniques mentioned above, the MRI, which is a nondestructive probing technique, has its innate advantages for observing the migration pathway geometry (Luo et al., 2004, 2008) and measuring oil saturation within the 3D migration path (Miao et al., 2004). It can directly detect fluids and their flow pattern through the pores (Koptyug et al., 2002), and is not affected by the shape, transparency and the refraction index of the media (Wang et al., 1996). Mandava et al had used the MRI to observe two-phase flow and estimate the fluid saturation in porous media in 1990. And Wang et al. (1996) had proved that the MRI is a suitable method to evaluate the residual oil saturation in cores. Fukushima (1999) introduced the MRI to observe fluid velocity field, diffusion processes, and modification of the porous medium. Koptyug et al. (2002) applied the MRI to study gas flow and diffusion in a glass bead packing model. 
In this paper, we focused on the 3D effects of secondary oil migration and studied the lateral migration pathway characteristics through the physical experiments by designing a large 3D packing porous medium box-shaped model. Dyed oil was used as non-wetting phase to conduct the physical secondary migration experiments with the 3D box model. The MRI technique was applied to observe the migration pathway morphology and to measure oil saturation within the migration pathways. Some insightful outcomes including the lateral migration pathway thickness, which is in good agreement with theoretical value, and hydrocarbon migration morphology vertically and horizontally were obtained through this experiment measurements under the given condition of an impermeable top inclined cap and the limitation of the experimental settings.

The paper is organized as follows. In section 2, we introduce the 3D experimental model and the MRI observation method. In section 3, we describe experimental results qualitatively and quantitatively. In section 4 , we discuss the relation between characteristic migration pathway sizes and the Bond number, and the fractal characters of the lateral migration pathway at different scales. The last section is conclusion.

\section{Physical experiment preparation and observation methods}

\subsection{Sample preparation}

A plane-parallel 3D box model of $100 \times 40 \times 14 \mathrm{~cm}^{3}$ has been constructed (Fig. 1). The length of $100 \mathrm{~cm}$ was selected to ensure that oil can migrate laterally far enough without impacted by boundary limit; the horizontal width of $40 \mathrm{~cm}$ was based on the 
inner diameter of the MRI testing chamber which is about $50 \mathrm{~cm}$ wide; the height of $14 \mathrm{~cm}$ was chosen to make sure that the thickness of the lateral migration pathway is not affected by the size of the model and also to keep reasonable weight and size of the model as we had to move around the entire model for measurements. The material made of the box is one center meter $(1 \mathrm{~cm})$ thick plexiglass plate, selected because of the requirements of being a non-magnetic material for MRI and possessing a sufficient mechanical strength to hold the model. The base inlet plate and four sides of the box were stuck together directly with glue while the outlet plate (of dimensions $40 \times 14 \times 1 \mathrm{~cm}^{3}$ ) was left re-moveable so that the model can be filled with glass beads conveniently. The outlet plate and the sides were connected with nylon bolts and sealed with back-shaped rubber gasket under pressure during the experiment. The tubes for fluid flow were installed respectively in the inlet and outlet plates. The glass plate was used to avoid the oil migration pathway to be affected by a different wettability of the boundary compared to the porous medium (Thomas and Clouse, 1995).

Glass beads with the diameter of $0.4-0.6 \mathrm{~mm}$ were used in the experiment as porous medium. To make sure that they were water-wet, all glass beads used were carefully washed with acid and alkaline solutions and then heated to a temperature of $500^{\circ} \mathrm{C}$ for at least 30 minutes (Luo et al.,2004).

When filling the box with the glass beads, the 3D box model was first positioned vertically and filled with water. The outlet plate was removed and a special sieve was fastened on the top of the model. And then the mixture of glass beads and water was 
poured into the sieve and the glass beads deposited uniformly and continuously so that the porous medium was as homogeneous as possible. At the same time, the sides of the model were knocked with a rubber hammer around the model in a continuous and uniform way in order to make sure that the glass beads were closely packed. After filling the glass beads, the back-shaped rubber gasket and the outlet plate were placed onto the top of the model and bolted to seal the model carefully.

The permeability of the medium is $36.82 \mu \mathrm{m}^{2}$ and the radii of pores are in the range of 0.185 to $0.225 \mathrm{~mm}$, as measured by Hou et al. (2004). Once the model was entirely saturated with water (of density $\rho=1000 \mathrm{~kg} / \mathrm{m}^{3}$ ), it was then placed obliquely with a horizontal angle $(\alpha)$ of $36^{\circ}$ during the experiment (see Fig. 1). Dyed kerosene oil with a density ( $\rho$ ) of $778 \mathrm{~kg} / \mathrm{m}^{3}$ was injected into the model through the base inlet tube at the bottom by a micro pump at a constant rate of $0.1 \mathrm{ml} / \mathrm{min}$. Given this open design system, a normal pore pressure is expected within the box, and the excess water consequently was driven out of the box through the outlet tube. The interfacial tension between the oil and water was measured to be $\gamma=0.0289 \mathrm{~N} / \mathrm{m}$. Since oil was dyed in red, the movement of oil could be traced visually as well in the vicinity of the box limits.

\subsection{The MRI methods of observation}

The Wandong medical 1.5T superconducting MRI system was utilized to observe the migration process in the experiment. The presence of ${ }^{1} \mathrm{H}$ in both oil and water induces MRI signals while scanning through the migration pathway. Due to the molecular structural difference between water and oil, the relaxation rates of ${ }^{1} H$ in 
oil and water are different and, in fact, the relaxation rate of ${ }^{1} H$ associated to water molecules is quicker than that in oil. That is when water-soluble $\mathrm{Mn}^{2+}$ is added into water, the relaxation rate of ${ }^{1} H$ in water molecules fastens but the relaxation rate of ${ }^{1} H$ in oil remains unchanged (Chang et al., 1993; Wang et al., 1996). Therefore the $M n^{2+}$ $(0.7 \mathrm{~g} / \mathrm{L})$ solution could be added to water to distinguish the oil MRI signal from that of water. It was confirmed that the adding $\mathrm{Mn}^{2+}$ into water did not change the interfacial tension between water and oil.

Since the box model occupies a significant portion in the MRI testing chamber, the scanning parameter options were set as follows: the largest available magnetic coil and a FOV of $384 \times 384 \mathrm{~mm}^{2}$, corresponding to a slice resolution of $1.5 \mathrm{~mm}$. These scanning conditions imposed by the size of the model have impact on the resolution of the scanner because the image quality and the SNR (signal to noise ratio) decrease with the increase of both the coil size and the FOV (Mei et al., 2008). Therefore, an image noise reduction process (or a filter) is required to re-process the scanned images. After analyzing the signal distribution of the migration pathway, a "noise reduction filter" based on adjacent pixels was adopted as described here. First, the noise threshold $T_{h}$ was set as the maximum value of signal in the clear-of-oil areas near the migration pathway, and then a threshold concerning the 8 neighboring pixels in $2 \mathrm{D}$ space was applied with ensuring of oil present. This threshold $n_{k}$ was set to 3 . If the pixel gray value of images was smaller than $T_{h}$ the pixel was considered as a noise point and its gray value was set to 0 , In addition, the number $n$ of the adjacent pixels whose gray value was larger than $T_{h}$ was counted. If $\mathrm{n}$ was larger than $n_{k}$, the pixel 
was considered as a point covered by oil and its gray value was confirmed, otherwise it was considered as a noise point and its gray value was also set to 0 . Figure 2 shows the results of the migration pathway image before and after this noise reduction procedure. It demonstrated that the noise was filtered out almost completely from the background image while the characteristics of dominant migration pathway are preserved.

Another processing step needed is to eliminate the different image results because of the scans taken at different times. A linear transformation was applied to normalize all slice signals before reconstructing the 3D migration pathway.

Also, in order to measure and calibrate oil saturation value within the migration pathway from the MRI images, a small glass tube filled with $100 \%$ oil was placed along with the model and scanned too at the same time when scanning the migration path, and used it for the oil saturation calibration.

In a preliminary experiment, the longitudinal relaxation time (T1) and the transverse relaxation time (T2) of an oil-saturated glass bead packing similar to the model, were measured, using the inversion recovery method and echo spin method respectively (Tyryshkin, 2003). T1 was found to be $1011 \mathrm{~ms}$, and T2 to be $810 \mathrm{~ms}$. The Fast Spin Echo-Proton Density Weight Image (FSE-PDWI) sequence was selected here in this work to scan the migration pathway. According to the measured relaxation times from above, echo time (TE) and repetition time (TR) were set respectively to $108 \mathrm{~ms}$ and $9000 \mathrm{~ms}$. Accordingly, conditions for the spin density image, e.g. $-T R>>T 1$ and $T E<<T 2$ (Xiao et al., 1995), - were satisfied. The 
acquisition time of for each slice is proportional to TR and it was about 13.73 second in our experiment.

Under these given conditions, the MRI signal varies linearly as a function of the volumetric oil concentration. The oil saturation within the migration pathway can thus be quantitatively estimated from magnetic resonance images (Miao et al., 2004). The gray level of the image is just proportional to the proton density (in oil) and oil saturation in the migration pathway can be calculated as (Chen et al., 1992; Luo et al., 2004) below:

$$
s=y /(B \sqcap \varphi) \times 100 \%
$$

where $s$ is the oil saturation; $y$ is the gray level of the migration pathway; $B$ is the gray level of the calibration; and $\varphi$ is the porosity of the model, which is about 0.36 , calculated by measuring the total volume and the water volume filling the pore space in the glass tube.

During the scanning process, the thickness of the slice and the interval between the slices were respectively set to be $3.0 \mathrm{~mm}$ and $0.3 \mathrm{~mm}$. The resolution along the slice was $1.5 \mathrm{~mm} \times 1.5 \mathrm{~mm}$ (

This 3D physical experiment lasted about six days (150 hours) and the Table 1 gives the progressive steps of the experiment. While the model was placed obliquely at $36^{\circ}$ during oil migration, it was set horizontally when the migration pathway was being scanned by MRI, a constraint due to scanning device.

\section{Experimental results}

The experiment on secondary migration in our 3D physical model was carried out 
successfully. The migration process and pathway morphology were observed visually and recorded through MRI images and oil saturation within the migration path was computed based on the magnetic resonance images.

\subsection{Qualitative observation by visual inspection and by MRI}

\subsubsection{Results observed visually}

Since injected oil was dyed, the process of oil migration and the morphology of the oil pathway were initially observed by visual inspection. Because the model is not completely transparent through the glass bead column, the migration pathway along the vertical path could not be detected optically before the oil front had reached the impermeable top inclined cap. As soon as the oil front had reached the top inclined cap, oil started to move laterally under the top inclined glass plate. It was noted that the development of the migration pathway is not a continuous process but often occurs through suddenly jumps which are similar to the so-called Haines jumps (Morrow, 1970). The migration feature of such jumps was due to the motion of the oil migration front paused from time to time although the oil was continuously injected. While the front was stagnant, the pathway became thicker and therefore tended to develop transversely, and consequently new fingers emerged from this enlarged head. There were usually several fingers in the front of the lateral migration path and these fingers could merge or split from time to time. In some occasions, water was trapped in the migration pathway when two fingers, especially two non-adjacent fingers, coalesced. Figure 3 is a picture of the migration pathway taken by a digital camera. It shows that the migration pathway was meandering and very irregular in shape. The 
horizontal width of the migration cluster fluctuated drastically along the main direction of the lateral migration. The front of the fingers did not touch the edges on sides of the model horizontally which proved that oil could migrate transversely freely without any constraints because of the boundaries of the box model.

\subsubsection{Results observed by MRI}

\section{The whole migration pathway}

The entire 3D migration pathway was reconstructed using the MRI slices (Figure 4). Figure 4A presents the top view image to show the entire migration pathway morphology along the direction parallels to the top glass cap, showing that many small fingers developed along the main pathway and some water-saturated areas (e.g. migration shadows) were formed. Generally, the width of horizontal migration path decreased along the direction of lateral migration, and its mean value $(\xi)$ was measured to be about $8.36 \mathrm{~cm}$. Figure 4B is the lateral view image (vertical cross section of the model) to illustrate the change of thickness of the migration pathway along the lateral migration direction. It shows that the oil had migrated upwards first from the point of where injected into the model, and then changed its direction once the oil front reached the impermeable top inclined cap. The lateral migration pathway is a thin layer-shaped zone, paralleling to the top inclined cap with a generally stable vertical thickness $(H)$ of 2 to $4 \mathrm{~cm}$ in the lateral migration direction, under the given conditions of the 3D box geometry and experiment settings. A closer examination of the inside texture of the lateral migration path with nearly constant thickness, it was consisted by many small clusters of complex morphology embedded within the 
bounding volume paralleled to the top inclined impermeable cap, as could be observed when rotating the $3 \mathrm{D}$ image.

\section{Vertical migration pathway and snap-off}

The first movement of oil after injection in the model was upward driven by buoyancy. The vertical pathway was scanned several times using MRI after the front had reached the top cap. The shape of the vertical migration path was roughly cylindrical and there were many little fingers around the main pathway (Figure 5A). The vertical migration pathway shrank and snapped off obviously when the oil front had reached the location $53 \mathrm{~cm}$ away from the inlet plate and most of the pathway split into oil drops or small oil bands (Figure 5B). With continuing injection, it then was refilled and at the meanwhile the migration front moved further forwards (Figure 5C).

Compared with Figure 5B, the size of the vertical pathway in Figure 5C was increased, but it was still smaller than the size of the vertical pathway when the oil front had initially reached (Figure 5A). The initial lateral pathway shrank and snapped off at the beginning point of the lateral migration pathway (or the end of vertical migration) while the vertical pathway was refilled (see Figure 5C).

\section{Lateral migration front}

During the time period of oil migrating laterally, the migration pathway was scanned at the different locations along the lateral migration direction so that the $3 \mathrm{D}$ migration pathway could be reconstructed according to these scanned $2 \mathrm{D}$ slices (Figure 6), and oil migrating phenomena and behavior could be recorded clearly during the migration process. Figure $6 \mathrm{~A}$ and $6 \mathrm{~B}$ show respectively the $3 \mathrm{D}$ migration 
front when it was at the locations of $41 \mathrm{~cm}$ and $70 \mathrm{~cm}$ away laterally from the inlet plate. As shown in Figure 6A, new fingers were formed "suddenly" along the oil front once the migration front became thicker and started to move transversely. Figure 6B shows the coalescence of oil fingers and its consequent effect of the "trapped-area" where no oil had migrated into and left behind the migration front, as well as the splitting of fingers after coalescence.

\section{The lateral migration path evolution with time}

The migration pathway was also scanned repeatedly at the same location in order to observe the migration path development with time. Figure 7 shows the slices taken at the location of $30 \mathrm{~cm}$ away from the inlet plate at the moments when the lateral oil front arrived at the locations of $53 \mathrm{~cm}$ (Fig-7A), $70 \mathrm{~cm}$ (Fig-7B), $84 \mathrm{~cm}$ (Fig-7C) and the outlet point (Fig-7D). These slices shows that the overall morphology of the pathway remained almost the same during the lateral migration but the coalescences (Figure 7B and C) and snap-offs occurred locally (Figure 7A and D) within the 3D migration pathway.

\subsection{Quantitative observation by MRI}

\subsubsection{Size of the lateral migration pathway and oil saturation within pathway}

After the migration front had reached the top end of the model in the experiment, the entire migration pathway was scanned and oil saturation within the migration path was calculated according to the $2 \mathrm{D}$ MRI slices. In order to observe the $3 \mathrm{D}$ path size/shape and oil saturation distribution, a 2D slice was taken for every $10 \mathrm{~cm}$ along the lateral migration direction, starting from the location of $25 \mathrm{~cm}$ (167 pixels) away 
from the inlet plate of the model (Figure 8). It can be seen from Figure 8 that the thickness of the lateral migration pathway was stable about a constant value of 2-4 cm vertically in the Y direction. On contrary, the horizontal width (in the $\mathrm{X}$ direction) of the lateral migration pathway fluctuated dramatically along the lateral migration direction Z. The entire lateral migration pathway was not consisted of a single connected pore layer beneath the top inclined glass cap, but instead had many small fingers developed surrounding the main survived path and the ends of the upward fingers reached the glass cap. Figure 8 also shows that the oil saturation distribution was heterogeneous in the lateral migration pathway. The oil saturation within most area was between $40 \%$ and $60 \%$, and it could reach $80 \%$ only at a few points. Comparing the oil saturation within this $3 \mathrm{D}$ lateral migration path to the vertical migration pathways (Luo et al., 2004, 2008), the oil saturation distribution was not artificially affected by the glass cap in the lateral migration pathway. Figure 9 and 10 are plots of the sectional area of the lateral migration path and average oil saturation within the pathway, respectively, with the distance along the lateral migration (in $\mathrm{Z}$ direction). It can be seen that the sectional area of the lateral migration pathway fluctuated drastically with its maximum value of $14 \mathrm{~cm}^{2}$ and minimum of only $1 \mathrm{~cm}^{2}$ but the average oil saturation remains relative stable about $47 \%$.

\subsubsection{Size of the migration pathway and oil saturation at different stages}

The previous research (Zhang et al., 2003; Luo et al., 2004) has noticed in their experiments that the oil saturation within pathway varies according to various migration stages, e.g. from $70-80 \%$ during the growing stage to $25-35 \%$ during the 
shrinking. Therefore both the migration pathway morphology and oil saturation within the pathway at the different stages were considered during this $3 \mathrm{D}$ physical experiment.

We had scanned the migration pathway at the location of $45 \mathrm{~cm}$ away from the inlet plate of the model at the various stages of oil injection - e.g. (1) during initial injection when first pathway formed , (2) during the residual oil stage after injection stopped, and (3) during re-injecting stage after the pause of injection. Figure 11 shows the results of the sectional area of the pathways and the average oil saturation in the pathways. The sectional area of residual pathway was significantly smaller than it when migration pathway initially formed. The sectional area was increased again when the oil re-injected, but it was still smaller than the initial stage.Although oil saturation with initial injection is the largest and the re-injecting stage is still larger than it in the residual stage, the differences of oil saturation among different stages are not large.

\section{Discussion}

Aforementioned MRI images show that the lateral migration pathways beneath an impermeable top cap appear as a sheet like shape with a certain thickness. That seems like those formed in 2D Hele-Shaw equipment (Hou et al., 2005; Yan et al., 2011) and different from those expected in 3D space (Thomas and Clouse, 1995). Thesefore, it is necessary to discuss the agreement between theory and measurements for the morphology of the migration pathways.

Previous studies (Tukugaga et al., 2000; Luo et al., 2004; Luo, 2011) indicate that 
the dimensionless Bond number (Bo) (Wilkinson, 1984, 1986), describing the relationship between buoyancy and interfacial forces at the scale of pore, provides a suitable characterization of the migration pathway. In our experiment, $B o$ can be estimated as:

$$
B o=\frac{\Delta p_{\text {grav }}}{\Delta p_{\text {int }}}=\frac{\Delta \rho g a^{2} \sin \alpha}{\gamma}
$$

Where $\Delta p_{\text {grav }}$ and $\Delta p_{\text {int }}$ are hydrostatic pressure difference and interfacial pressure difference respectively, $\rho=222 \mathrm{~kg} / \mathrm{m}^{3}$ is the density difference of the oil and water, $g$ $=9.8 \mathrm{~m} / \mathrm{s}^{2}$ is the gravity acceleration, $a=4.1 \times 10^{-4} \mathrm{~m}$ is the mean pore diameter, $\gamma=0.0289 \mathrm{~N} / \mathrm{m}$ is the interface tension between oil and water. So $B o=7.44 \times 10^{-3}$. In contrast with the vertical migration pathway, the lateral migration pathway can be assimilated to a $2 \mathrm{D}$ one because the thickness of the lateral migration pathway remained stable to $2-4 \mathrm{~cm}$. The vertical extent $(V)$ of the lateral migration pathway can be scaled as:

$$
V \approx a / B o
$$

due to the balance between the buoyancy force and capillary force. The thickness perpendicular to the inclined cap is thus:

$$
H=V \cos \alpha \approx a \cos \alpha / B o
$$

So $H$ is expected to be around $5 \mathrm{~cm}$. The measured lateral migration pathway thickness, between 2 and $4 \mathrm{~cm}$ is in rough agreement with the theoretical value. These results suggest that the $3 \mathrm{D}$ experimental lateral migration study can be divided into two subsets of $2 \mathrm{D}$ experiments. One is to study the thickness of the migration pathway when the 2D model is placed obliquely and vertically, as used in Thomas and Clouse's 
experiment (1995); the other is to study the morphology of the migration pathway under the impermeable cap when the 2D model is placed obliquely and horizontally, as used in Birovljev et al's study (1991).

The snap-off effect was found both in the vertical migration pathway and in the lateral one. The refilling of the vertical migration pathway after the snap-off and the alternating of coalescence and splitting meant there may be cyclical changes in the migration pathway. However this phenomenon appeared to occur more frequently in the vertical migration pathway than in the lateral one. This may be due to the interplay between local pinning forces and buoyancy leading to the snap-off of the migration pathway (Wagner and Birovljev et al., 1995, 1997) and the effect of the buoyancy in the vertical migration pathway was stronger than in the lateral migration pathway.

The fractal dimension was measured using box counting method. Before fractal dimension computation, the slices were filtered using the method mentioned in section 2 and transformed into binary images. The lateral migration pathway is covered with boxes with side containing $K$ pixels. The box is empty if it covers none of the migration pathway. The number $N$ of non-empty boxes is counted. A series of $\mathrm{N}$ is obtained as function of gradually changing choices of $K$ and points $\left(K_{i}, N_{i}\right)$ are drawn in double logarithm coordinate system (Figure 12). The fractal dimension is equal to the minus slope of the linear section of the fitting line between the box size and the box number. As shown in Figure 12, the fitting line can be divided into three sections. At small scale (section 1, from 1 pixel to 11 pixels), the migration pathway was dominated only by the porous disorder, and the fractal dimension is $2.2 \pm 0.3$, 
which is in rough agreement with the value 2.50 expected for capillary fingering, consistent with three dimensional invasion percolation models (Wilkinson, 1986). At medium scale (section 2 on Figure 12), at scales between $H$ and $\xi$, the migration pathway is essentially a thick plate parallel to the cap, i.e. it is dominated by the porous disorder and the buoyancy at the same time, and the fractal dimension is around 1.9. Up to the large error bar inherent to the narrow scale range in this analysis, this is both compatible with a large scale (larger than $H$, section 3 on Figure 12), the migration pathway was dominated only by the buoyancy, looks like an upwards linear structure, which is reflected by its fractal dimension which is almost 1 . The separation scale $W l=2.25 \mathrm{~cm}$ between sections 1 and 2 (left dash-dotted line on Figure 12) is close to the vertical thickness of the lateral migration pathway $H \sim 2-4 \mathrm{~cm}$. The second separation scale $W 2$ between sections 2 and 3 is $9.9 \mathrm{~cm}$ (right dash-dotted line on Figure 12), which is close to the mean width $\xi$ of the lateral migration pathway.

The relationship between injected and MRI measured oil volume is now discussed. With the average oil saturation in migration pathway of each slice and the sectional area of migration pathway, the oil volume within such slice can be calculated as:

$$
v_{s}=\bar{S}_{s}^{*} A_{s} * T * \varphi
$$

in which, $\bar{S}_{s}$ is the average oil saturation of such splice in migration pathway, $A_{s}$ is the sectional area of migration pathway, $T$ the splice thickness of the slice. Though the part between two splices can not be MRI scanned, since the interval between splices is much smaller than the splice thickness, the oil volume in the gaps between splices can be interpolated from the two neighboring slices: 


$$
v_{G}=\frac{\left(A_{s}+A_{s+1}\right)}{2} * \frac{\left(\overline{S_{s}}+S_{s+1}^{-}\right)}{2} * G * \varphi
$$

where $n$ is the total number of total splices and $G$ is the gap between the two neighboring slices. So the oil volume in the whole model is obtained as:

$$
v_{o}=\sum_{s=1}^{n} V_{s}+\sum_{G=1}^{n-1} V_{G}
$$

The oil volume computed according to slice oil saturation and area is $82.17 \mathrm{ml}$. On the other hand, the actually injected oil volume is computed by multiplying the injection rate by the injection time, which is $96.4 \mathrm{ml}$. The computed oil volume is slightly less than the actually injected oil volume. It can be explained by two reasons. One is the pure oil is used as the calibration, which magnetic resonance signal relax slower than the oil in the pores, that induces the calculated saturation is less than the actual one. The other is the smallest connected features of the migration pathway may be suppressed during slice filtering.

Accurate estimates of the losses occurring during secondary oil migration are a vital part of valid exploration assessments (Sylta, 1993; Lewen et al., 2004; Luo et al.., 2004, 2007, 2008). Secondary oil migration is a very complex process (McNeal, 1961; Harms, 1966; Berg, 1975; Schowalter, 1979). The ratio between migration pathway and the carrier is small, but it can change from $1 \%$ and $10 \%$ (Schowalter, 1979; England et al., 1987; Dembicki and Anderson, 1989; Catalan et al., 1992), which makes the estimates of residual oil saturation from different researchers are not consistent (Schowalter, 1979; England et al., 1987; Dembicki and Anderson, 1989; Hirsch and Thompson, 1995) and makes the accurate estimates of the losses occurring 
during secondary migration difficult. So Carruthers and Ringrose(1998) pointed out that determining the oil-rock contact volumes is the basic work for accurate estimates of the losses occurring during secondary oil migration. Combined with our study, oil-rock contact volumes can be evaluated based on the thickness and the width of pathway during the lateral migration process. Knowing the oil saturation distribution, the oil losses can be estimated easily. While oil saturation in the residual pathway was slightly lower than that obtained when the pathway was forming, the residual migration pathway size shrinks dramatically and the section area of the residual migration pathway is about $30 \%$ of the initial pathway. So shrinking of the pathway after stopping injection appears as the main reason for the occurrence of small losses during the secondary oil migration.

\section{Conclusions}

Compared to the cylindrical shape of the vertical migration pathway, the lateral migration pathway was a layer-shaped zone under and parallel to the impermeable inclined cap. Under the given experimental conditions, the thickness of the lateral migration pathway, perpendicularly to the impermeable cap, was around a constant value between 2 and $4 \mathrm{~cm}$ while its horizontal width measured perpendicular to the slope of the cap fluctuated drastically.

MRI was successfully used to observe the morphology of the secondary migration pathway in this $3 \mathrm{D}$ experiment, to measure the size of the pathway and the oil saturation within the path. Oil saturation was heterogeneous in the lateral migration pathway but was almost not affected by the cap; oil saturation of most regions was 
between $40 \%$ and $60 \%$, and only at a small amount of points it could reach $80 \%$; the average oil saturation was stable at $47 \%$ following the lateral migration direction.

Dominated by porous disorder, buoyancy, or the interplay between them, the mass fractal dimension of the lateral migration pathway was measured to be between 2 and 2.5 at scales smaller than the thickness $H$, consistently with 3D capillary fingering, around 1.9 at scales between the pathway thickness $H$, consistently with 2D capillary fingering or fully two dimensional structures and its width $\xi$, consistently with the 2D capillary fingering or fully two dimensional structures, and around 1 above $\xi$, corresponding to a linear uprising geometry of the pathway.

\section{ACKNOWLEDGEMENTS}

This study was supported mainly by the Chinese National Science Foundation (40772090, 40902041), and partly sponsored by BHP Billiton (L1295XV00) and the University of Strasbourg, France. 


\section{Reference}

Berg R. R., 1975, Capillary pressure in stratigraphic traps, AAPG Bulletin, v. 59 no. 6, p. 939-959.

Birovljev A., and Wagner G., Meakin P., Feder J., and Jøssang T., 1995, Migration and fragmentation of invasion percolation clusters in two-dimensional porous media, Physical Review E, v. 51, no. 6, p. 5911-5915.

Carruthers D., and Ringrose P., 1998, Secondary oil migration: oil-rock contact volume, flow behaviour and rates: In. Parnell J.(ed.) Dating and duration of fluid flow and fluid-rock interaction, Geological Society, London, Special Publications, v. 144, p. 205-220.

Catalan L, FU W. F., Chatzis I., Francis A., and Dullien L., 1992, An experimental study of secondary oil migration, AAPG Bulletin, v. 76, no. 5, p. 638-650.

Chang C.T., MAndava S., Watson A.T., Sarkar S., and Edwards C.M., 1993, The use of agarose gels for quantitative determination of fluid saturations in porous media, Magnetic Resonance Imaging, v. 11, no. 5, p.11, 717-725.

Chen S., Kim K. H., Qin F., and Watson A. T., 1992, Quantitative NMR imaging of multiphase flow in porous media, Magnetic Resonance Imaging, v. 10, no. 5, p. 815-826.

Dembichi H. J., and Anderson M. J., 1989, Secondary migration of oil: Experiments supporting efficient movement of separate, buoyant oil phase along limited conduits, AAPG Bull., v. 73, no. 8 , p. 1018-1021.

Emmons W. H., 1921, Experiments on accumulation of oil in Sands, AAPG Bulletin, v. 5, p. 103-104.

England W. A., Mackenzie A. S., Mann D. M., and Quigley T. M., 1987, The movement and entrapment of petroleum fluids in the subsurface, Journal of the Geological Society, v. 144, 
no. 2 , p. $144,327-347$.

Fukushima E., 1999, Nuclear magnetic resonance as a tool to study flow, Annual Review of Fluid Mechanics, v. 31, p. 95-123.

Hindle A. D., 1997, Petroleum migration pathways and Charge Concentration: A three-dimensional model, AAPG Bull., v. 81; no. 9, p. 1451-1481.

Hirsh G. J., and Thompson A. H., 1995, Minimum saturations and buoyancy in secondary migration, AAPG Bull., v. 79, no. 5, p. 696-710.

Hou J., Li Z. Q., Zhang S.K., Cao X.L., Du Q.J., and Song X.W., 2009, Computerized tomography study of the microscopic flow mechanism of polymer flooding, Transp. Porous Med, v. 79, no. 3, p. 407-418.

Hou P., Luo X. L., Zhou B., and Zhang N. X.., 2005, An experimental study of episodic oil migration, Xinjiang Petroleum Geology, v. 26, no. 1, p. 33-35.

Koptyug I. V., Matveev A. V., and Vltobelli S. A., 2002, NMR studies of Hydrocarbon gas flow and dispersion, Applied Magnetic Resonance, v. 22, no. 2, p. 187-200.

Lenormand R., Zarcone E., and Touboul E., 1988, Numerical models and experiments on immiscible displacements in porous media, Journal of Fluid Mechanics, v. 189, p. 165-187.

Lewen M. D., Henry M. E., Higley D. K., and Pitman J. K., 2002, Material-balance assessment of New Albarny Chesterian petroleum system of the Illinois basin, AAPG Bull., v. 86, no. 5, p. 745-778.

Løvoll, G., Méheust Y., Toussaint R., Schmittbuhl J. and Måløy K.J., 2004, Growth activity during fingering in a porous Hele Shaw cell, Phys. Rev. E., v. 70, 026301, doi:10.1103/PhysRevE.70.026301. 
Løvoll, G., Jankov M., Måløy K.J., Toussaint R., Schmittbuhl J., Schäfer G. and Méheust Y., 2010, Influence of viscous fingering on dynamic saturation-pressure curves in porous media, Transport in Porous Media, v. 86, no 1, p. 305-324, doi: 10.1007/s11242-010-9622-8

Luo X. R., Zhang F. Q., Miao S., Wang W., Huang Y., Zhou B., Loggia D., and Vasseur G., 2004, Experimental verification of oil saturation and loss during secondary migration, Journal of Petroleum Geology, v. 27, no. 3, p. 241-251.

Luo X. R., Zhou B., Zhao S. X., Zhang F. Q., and Vasseur G., 2007, Quantitative estimates of oil losses during migration, Part I: the saturation of pathways in carrier beds, Journal of Petroleum Geology, v. 30, no. 4, p. 375-387.

Luo X. R., Yan J. Z., Zhou B., Hou p., Wang W., and Vasseur G., 2008, Quantitative estimates of oil losses during migration, Part II: measurement of residual oil saturation in migration pathways, Journal of Petroleum Geology, v. 31, no. 2, p.179-190.

Luo X. R., 2011, Simulation and characterization of pathway heterogeneity of secondary hydrocarbon migration, AAPG Bull., v. 95, no. 6, p. 881-898.

Mandava S. S., Ted Watson A., and Carl M. E., 1990, NMR imaging of saturation during immiscible displacements, AIChE, v. 36, no. 11, p. 1680 - 1686.

McNeal R. P., 1961, Hydrodynamic entrapment of oil and gas in Bisti field, San Juan County, New Mexico, AAPG Bull., v. 45, no. 3, p. 315-329.

Meakin P., Wagner G., Vedvik A., Amundsen H., Feder J., and Jossang T., 2000, Invasion percolation and secondary migration: Experiments and simulations, Marine and Petroleum Geology, v. 17, no. 7, p. 777-795.

Mei L. X., Wang H., and Li G. L., 2008, A two-channel surface coil for vertebral imaging on 
low-field MRI systems, Chinese Journal of Magnetic Resonance, v. 25, no. 1, p. 33-38.

Miao S., Zhang F. Q., Li T. J., Luo X. L., and Hou P., 2004, Application of NMR imaging technique to quantitative observation and analysis on hydrocarbon migration pathway, Acta Petrolei Sinica, v. 25, no. 3, p. 44-47.

Morrow N. R., 1970, Physics and Thermodynamics of Capillary Action in Porous Media, Industrial and Engineering Chemistry, v. 62, no. 6, p. 32-56.

Schowalter T. T., 1979, Mechanics of secondary hydrocarbon migration and entrapment, AAPG Bull., v. 25, no. 1, p. 723-760.

Seright R.S., Liang J., Lindquist W.B., and Dunsmuir, 2002, Characterizing disproportionate permeability reduction using synchrotron X-ray computed microtomography, SPE Reservoir Evaluation \& Engineering, paper number: SPE 71508.

Selle O. M., Jensen J. I., , Øyvind S., Andersen, T., Nyland B., and Broks, T.M., 1993, Experimental verification of low-dip, low-rate, two-phase (secondary) migration by means of X-ray absorption, Geofluids, Contributions to an international conference on fluid evolution, migration and interaction in rocks, Torquay (England), p. 72-75.

Sharma B. C., Brigham W., and Castanier L., 1997, CT Imaging techniques for two-phase and three-phase in-situ saturation measurements, Stanford University, 114p.

Stöhr M., Roth K., and Jähne B., 2003, Measurement of 3D pore-scale flow in index-matched porous media, Experiments in Fluids, v. 35, no. 2, p. 159-166.

Thomas M. M., and Clouse J. A., 1995, Scaled physical model of secondary oil migration, AAPG Bull., v. 79, no. 1, p. 19-29.

Toussaint, R., Løvoll G., Méheust Y., Måløy K.J. and Schmittbuhl J., 2005, Influence of pore-scale 
disorder on viscous fingering during drainage, Europhys. Lett., v. 71, p. 583, doi:10.1209/ep1/i2005-10136-9.

Turmer M.L., Knüfing L., Arns C.H., Sakellariou A., Senden T.J., Sheppard A.P., Sok R.M., Limaye A., Pinczewski W.V., and Knackstedt M.A., 2004, Three-dimensional imaging of multiphase flow in porous media, Physica A, v. 339, p. 166-172.

Tokunaga T. and Mogi K., Matsubara O., Tosaka H., and Kojima K., 2000, Buoyancy and interfacial force effects on two-phase displacement patterns: an experimental study, AAPG Bull., v. 84, no. 1, p. 65-74.

Tyryshkin A.M., Lyon S. A., Astashkin A. V., and Raitsimring A. M., 2003, Electron spin relaxation times of phosphorus donors in silicon, Phys. Review B, v. 68, no. 19, art no.193207.

Wagner G., Birovljev A., Feder J., and Jøssang T., 1997, Fragmentation and migration of invasion percolation clusters: Experiments and simulations, Physical Review E, v. 55, no. 6, p. 7015-7029.

Wang W. W., Lang D. J., and Lie W., 1996, The application of NMR imaging to the studies of enhanced oil recovery in China, Magnetic Resonance Imaging, v. 14, no. 5, p. 951-953.

Xiao L.Z., Du Y. R., and Ye C. H., 1995, NMR spin density micro-imaging for rock samples, Acta Physico-Chimica Sinaca, v. 11, no. 3, p. 196-198.

Zhang F.Q., Luo X. L., Miao S., Wang W. M., Zhou B., and Huang Y. Z., 2003, The patterns and its effects factors of secondary hydrocarbon migration: Petroleum Geology \& Experiment, v. 25, no. 1, p. 69-75.

Wilkinson D. 1984, Percolation model of immiscible displacement in the presence of buoyancy 
forces, Physical Review A, v. 30, no. 1, p. 520-531

Wilkinson D. 1986, Percolation effects in immiscible displacement, Physical Review A, v. 34, p.1380-1391. 


\section{Authors' CV:}

Jianzhao Yan $\sim$ Key Laboratory of Petroleum Resources Research, Institute of Geology and Geophysics, Chinese Academy of Science, Beijing, China, 100029; yjzh27@163.com

Jianzhao Yan is a post-doctor at the Institute of Geology and Geophysics, Chinese Academy of Science, Beijing. He received B.S. and M.S. in petroleum engineering and development from China University of petroleum, and a Ph.D in petroleum geology the Institute of Geology and Geophysics, Chinese Academy of Science. His interests now include theoretical and experimental analyses of secondary hydrocarbon migration, enhancement oil recovery, and the usage of NMR in petroleum industry.

Xiaorong Luo $\sim$ Key Laboratory of Petroleum Resources Research, Institute of Geology and Geophysics, Chinese Academy of Science, Beijing, China, 100029; $\underline{\text { luoxr@mail.iggcas.ac.cn }}$

Xiaorong Luo is a research scientist in the Chinese Academy of Sciences and has a B.S. and M.S. degree in geology from Northwestern University, China, and a Ph.D in geophysics from the University of Montpellier, France. His research interests focus on experimental and numerical modeling, geopressuring, and hydrocarbon migration and accumulation.

Weimin Wang $\sim$ School of Electronics Engineering and Computer Science, Peking University, Beijing, China, 100871; wmw@263.net.cn

Weimin Wang, Ph.D in radio physics, professor of the School of Computer Science and Electronics engineering Peking university. He has been working on the research of MR imaging in porous media since 1997. His research covers on the imaging method, hardware design and imaging processing of MRI. 
Renaud Toussaint IPGS, CNRS, University of Strasbourg, 5 rue Descartes, Strasbourg, France, 67000; renaud.toussaint@unistra.fr

Renaud Toussaint is a CNRS researcher in Geophysics at IPGStrasbourg working on fluid mechanics in porous media, and flow and fracture of mixtures of fluids and granular solids. He studied Mathematics, Theoretical Physics (Master in ENSLyon, agregation of mathematics), Geophysics (Master in IPGParis, PhD at University of Rennes I) and developed during his postdocs models of complex flows in the Physics Departments of the Universities of Oslo and Trondheim.

Jean Schmittbuhl IPGS, CNRS, University of Strasbourg, 5 rue Descartes, Strasbourg, France, 67084; Jean.Schmittbuhl@unistra.fr

Jean Schmittbuhl is a CNRS senior scientist at IPG Strasbourg (since 2004). He has a Master degree in Geosciences from ENS Paris and in Physics from Univ. Paris 6, an Aggregation in Geology, a PhD in Physics from Univ. Paris 6 (1994), an Habilitation in Geophysics (Univ. Paris 7 , 2001). His main research interest is in physics of geological processes (e.g. fluid flow, fracture, friction) combining experimental and numerical approaches.

Guy Vasseur SISYPHE, UMR7619, University Pierre et Marie Curie, Paris Cedex 05, France, 75252; guy.vasseur@upmc.fr

Guy Vasseur received an engineering degree from Ecole Polytechnique (Paris) in 1963 and a Doctorat d'Etat degree (PhD) from the University of Paris in 1971. He then became a senior scientist of Centre National de la Recherche Scientifique and worked at the Universities of Montpellier2 and Paris6 in various domains of geophysics. His main domain of competence is the modeling of heat and mass transfer in the earth crust. 
Fang Chen $\sim$ State Key Laboratory of Magnetic Resonance and Atomic and Molecular Physics, Wuhan Institute of Physics and Mathematics, Chinese Academy of Sciences, Hubei, Wuhan, China, 430071; chenfang040@sina.com

Fang Chen received his Ph.D degree in NMR from Wuhan Institute of Physics and Mathematics, the Chinese Academy of Sciences in 2004, and he has held a senior engineer position in this Institute since 2010. From 2004 to 2010, He served as a MRI R\&D Engineer in Beijing Wandong Medical Equipment Co. Ltd. His research interests include NMR, MRI, and porous materials studies.

Alan Yu BHP Billiton Petroleum, Geoscience Specialist Group, 1360 Post Oak Blvd., Houston, Texas 77560; Alan.A.Yu@,bhpbilliton.com

Alan Yu specializing in integrated petroleum system analysis is currently the Principal Geologist with BHP Billiton Petroleum. He earned his B.S. (1982) in petroleum geology from the Northeastern Petroleum University, China, and Ph.D. (1992) in basin modeling at the University of South Carolina (USC). After a two-year Post-doc. research in USC, Alan joined the industry in 1994 and has since working on petroleum E\&P supports in the worldwide basins including GOM, S. America, Africa, Canada, SE Asia and Australia for pre-drill predictions of hydrocarbon charge, fluid quality, pore pressure, top seal integrity and capacity, sub-salt sediment seismic velocity as well as reservoir quality. Alan has published over 30 papers in the related areas and is a member of AAPG since 1990.

Likuan Zhang $\sim$ Key Laboratory of Petroleum Resources Research, Institute of Geology and Geophysics, Chinese Academy of Science, Beijing, China, 100029; zhanglikuan1979@163.com Likuan Zhang is a postdocral researcher at the institute of Geology and Geophysics, Chinese 
Academy of Sciences. He received a B.S. degree in petroleum geology from Jilin University, China, and a Ph.D from the Institute of Geology and Geophysics, Chinese Academy of Sciences.

His research interests concern fluid flow in fault zones, numerical basin modeling, and hydrocarbon migration in petroleum systems. 
Figure 1. The sketch of the box model

Figure 2. The contrast of image before and after noise reduction

Figure 3. Picture of the lateral migration pathway

Figure 4. The whole 3D migration pathway

(A is the top view image to show the entire lateral migration pathway morphology, B presents lateral view image to show the change of the thickness of the lateral migration pathway.)

Figure 5. The 3D vertical migration pathways at different time

(The dot line indicates the location of the impermeable cap and the arrow indicates the lateral migration direction. A. when the front just reached the cap, the vertical migration pathway was roughly cylindrical; B. when the front migrated laterally, the vertical migration pathway snapped off obviously; C. the migration pathway was refilled.)

Figure 6. The 3D migration front of lateral pathway at different locations

(Oil migrated along the $\mathrm{Z}$ axis direction. A shows that new fingers formed suddenly along the front; B emphasizes the splitting of fingers after coalescence.)

Figure 7. The evolution of the lateral migration pathway along a slice $30 \mathrm{~cm}$ away from the inlet

Figure 8. Oil saturation distribution in the lateral migration pathway

Figure 9. Section area curve along the lateral $\mathrm{Z}$ migration direction

Figure 10. The average oil saturation within the migration pathway varies in the lateral $\mathrm{Z}$ migration direction

Figure 11. Characters of the migration pathways at different stages

Figure 12. The box number as a function of the box size 
(The fitting line can be divided into three linear sections corresponding to small, middle, and large scale respectively. $\mathrm{W} 1=2.25 \mathrm{~cm}$, is the separator between section 1 and section $2 ; \mathrm{W} 2=9.9 \mathrm{~cm}$, is the separator between section 2 and section 3.)

Table 1 the process of the experiment 
Table 1

\begin{tabular}{|c|l|}
\hline Time(hour) & \multicolumn{1}{|c|}{ Description } \\
\hline $0-3$ & $\begin{array}{l}\text { Oil migrated vertically and reached the glass cap; the vertical migration } \\
\text { pathway was scanned. }\end{array}$ \\
\hline $4-37$ & $\begin{array}{l}\text { Oil migrated laterally and breakthrough at the outlet plate; the lateral } \\
\text { migration pathway (trapped under the top cover) was scanned. }\end{array}$ \\
\hline $38-44$ & $\begin{array}{l}\text { Migration pathway evolved while oil was being injected and the } \\
\text { migration pathway was scanned. }\end{array}$ \\
\hline $45-121$ & $\begin{array}{l}\text { Oil migrated driven by buoyancy without oil being injected and the } \\
\text { residual migration pathway formed. }\end{array}$ \\
\hline $121-123$ & Residual migration pathway was scanned. \\
\hline $124-150$ & $\begin{array}{l}\text { Oil was injected into the model again and the migration pathway was } \\
\text { scanned. }\end{array}$ \\
\hline
\end{tabular}




\section{Figure. 1}

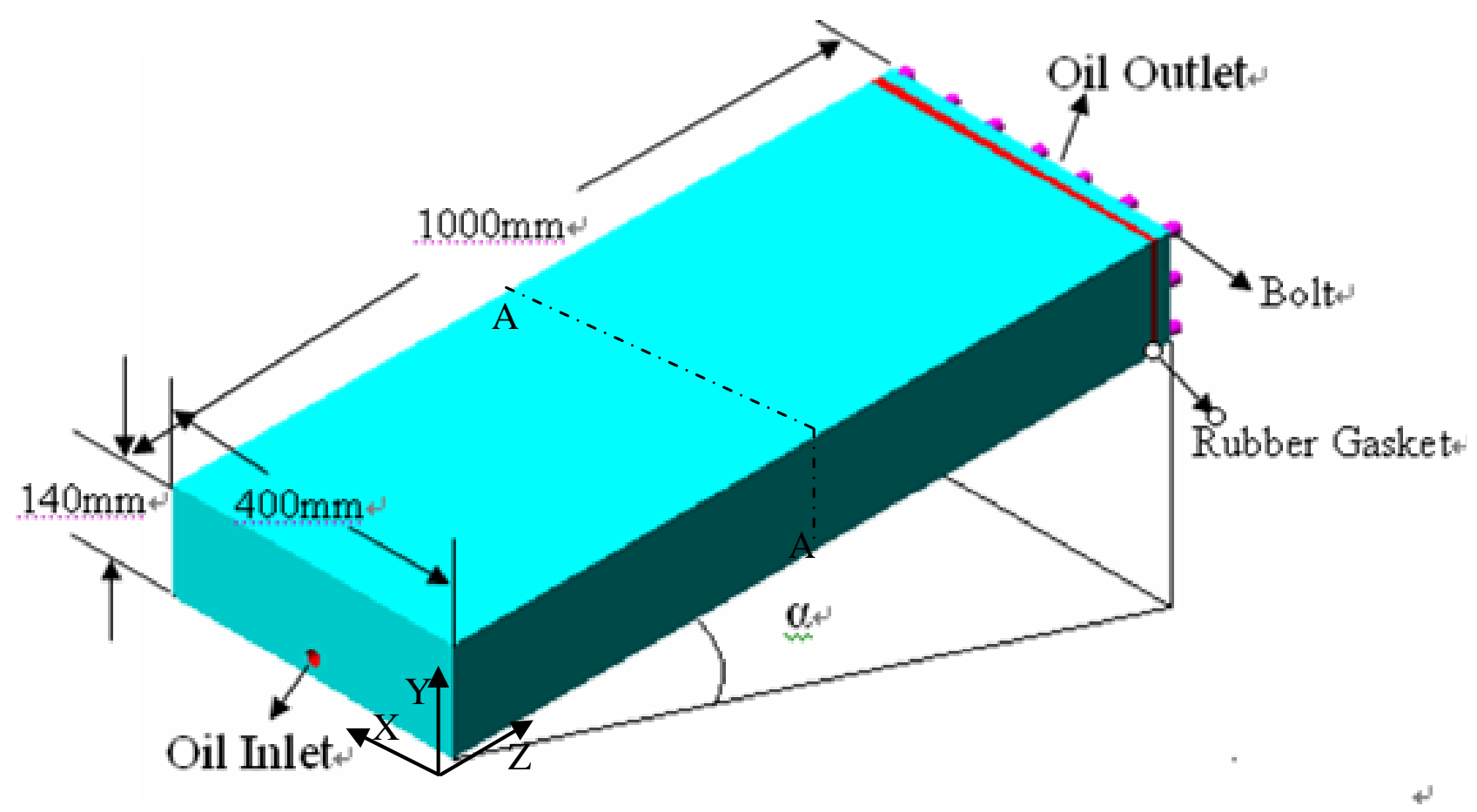

impermeable

glass cap

glass beads

plexiglass plate

A-A profile 


\section{Figure. 2}

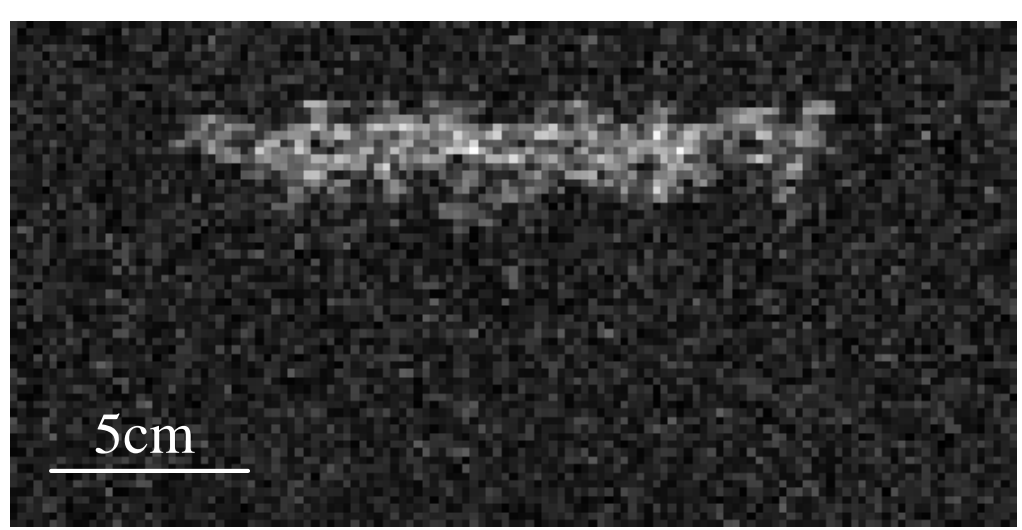

Iinitial image

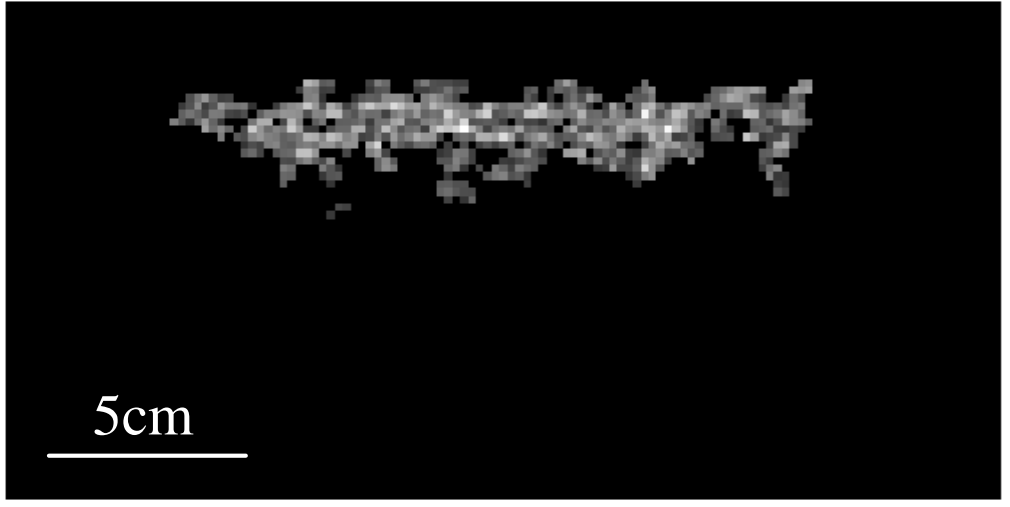

Filtered image 


\section{Figure. 3}

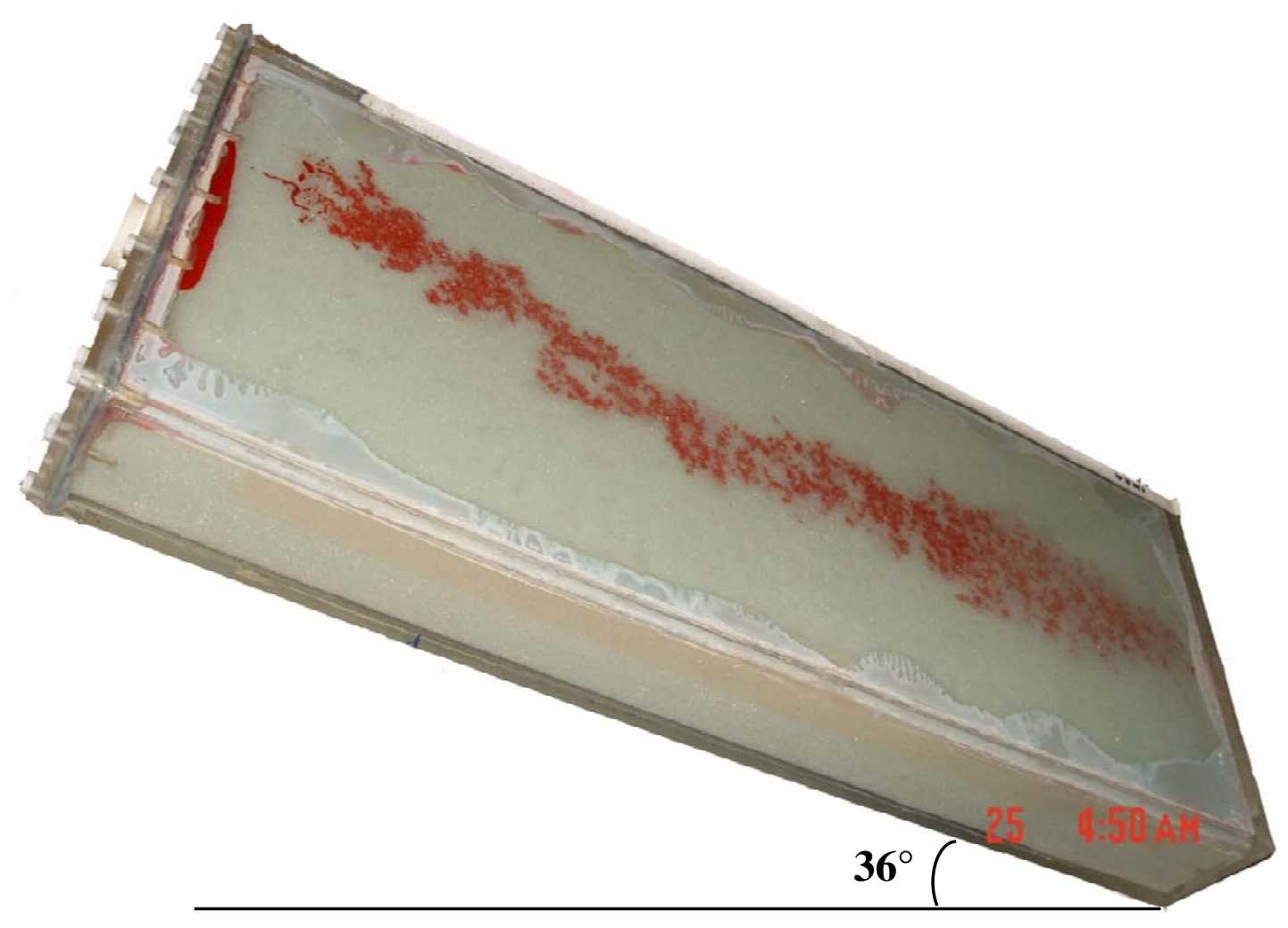




\section{Figure. 4}
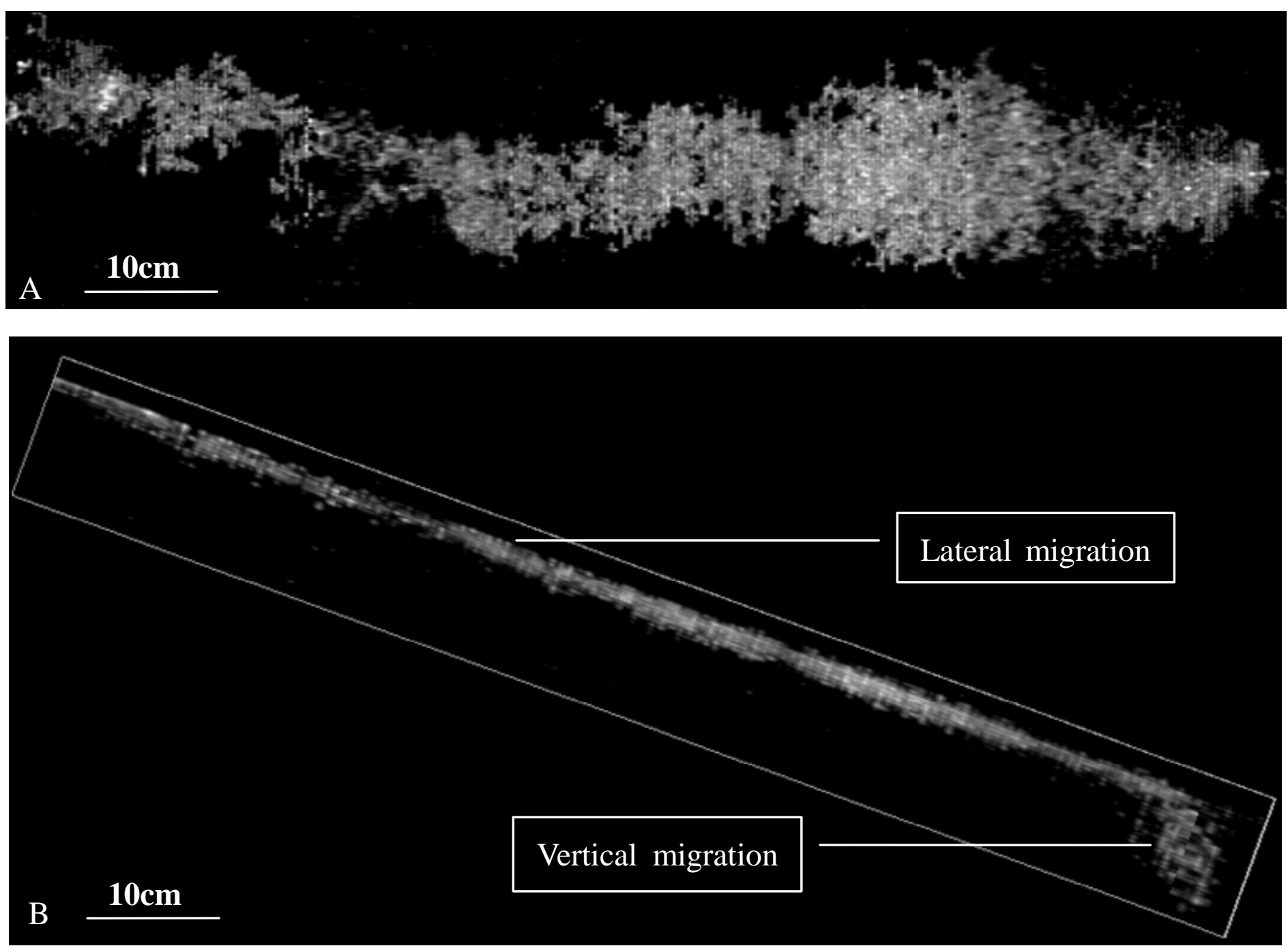


\section{Figure. 5}

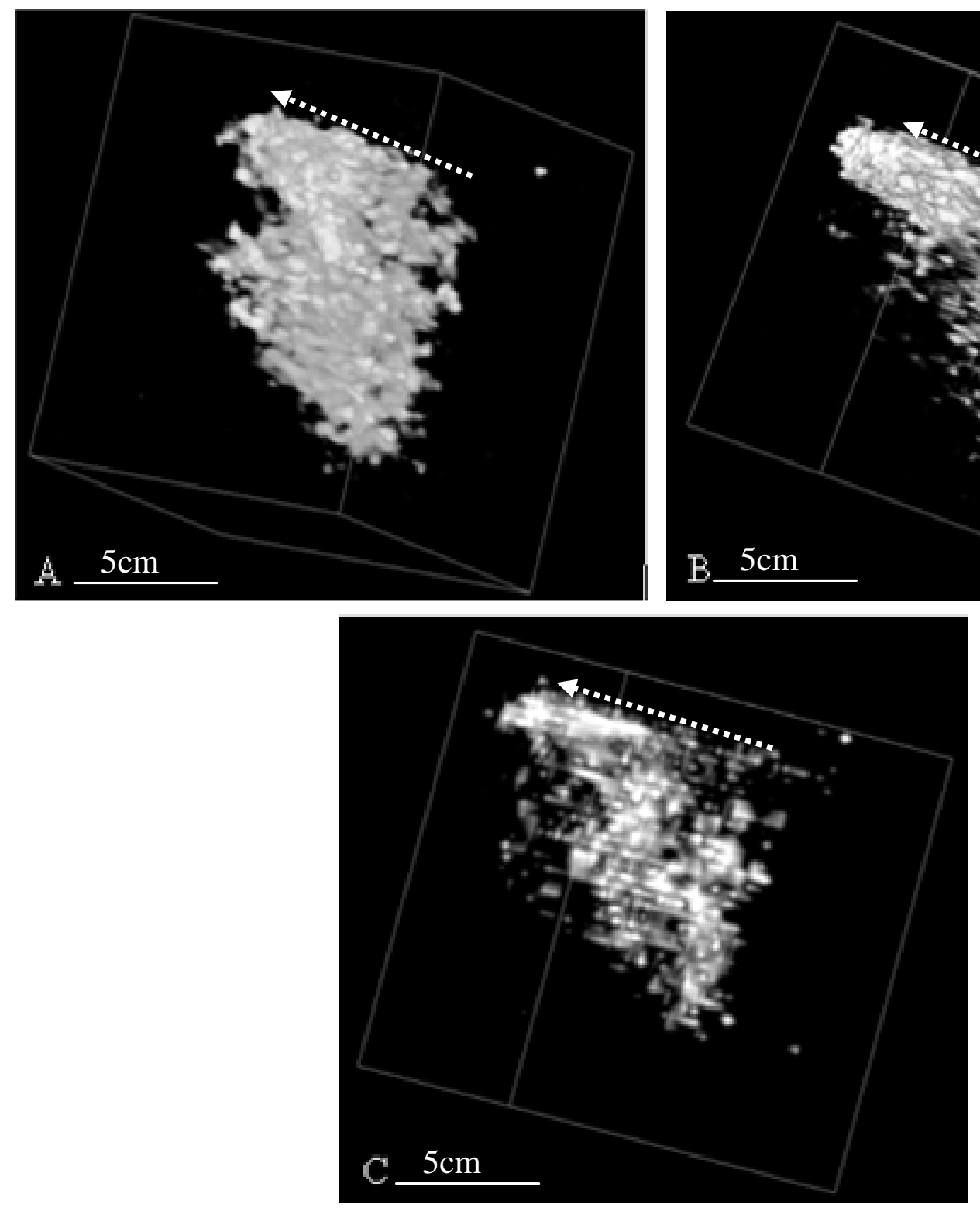




\section{Figure. 6}
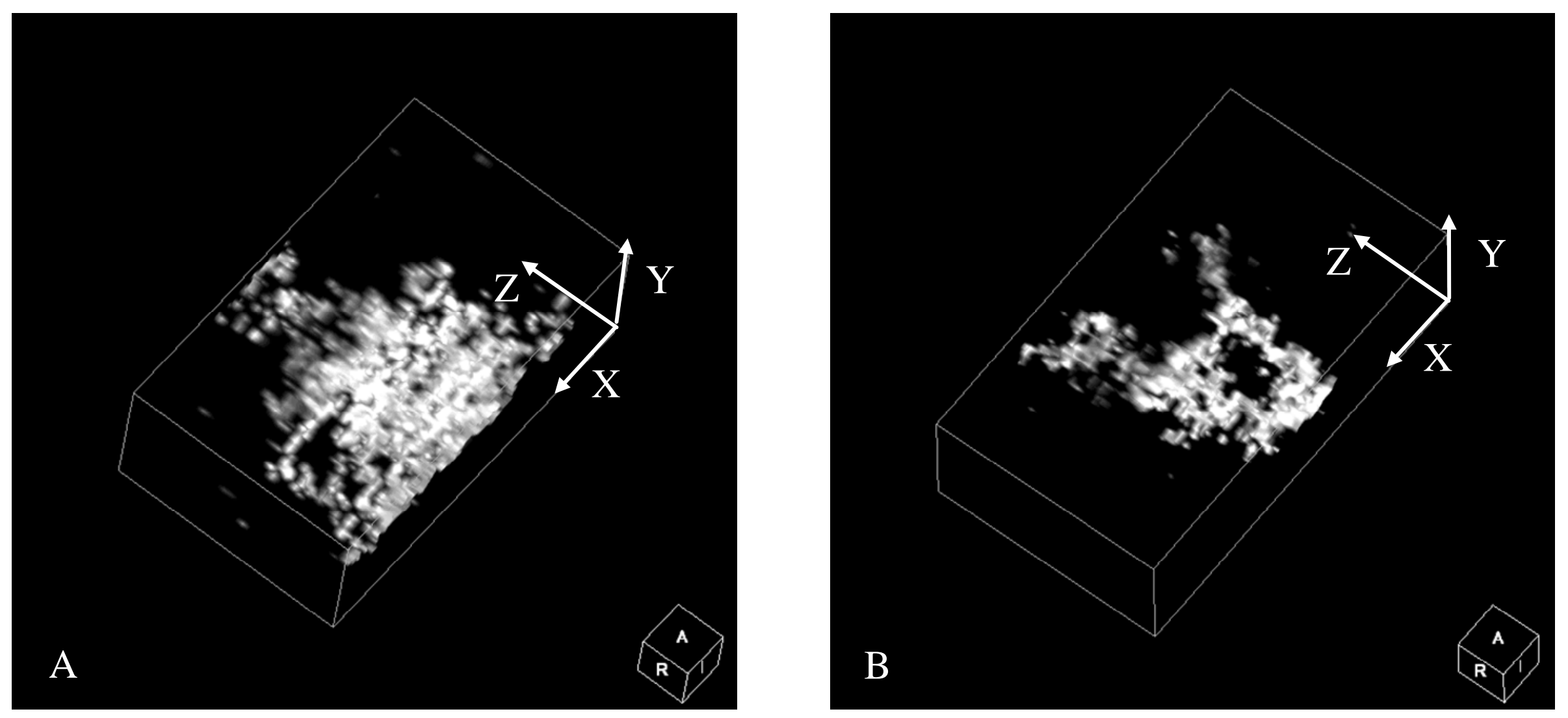


\section{Figure. 7}
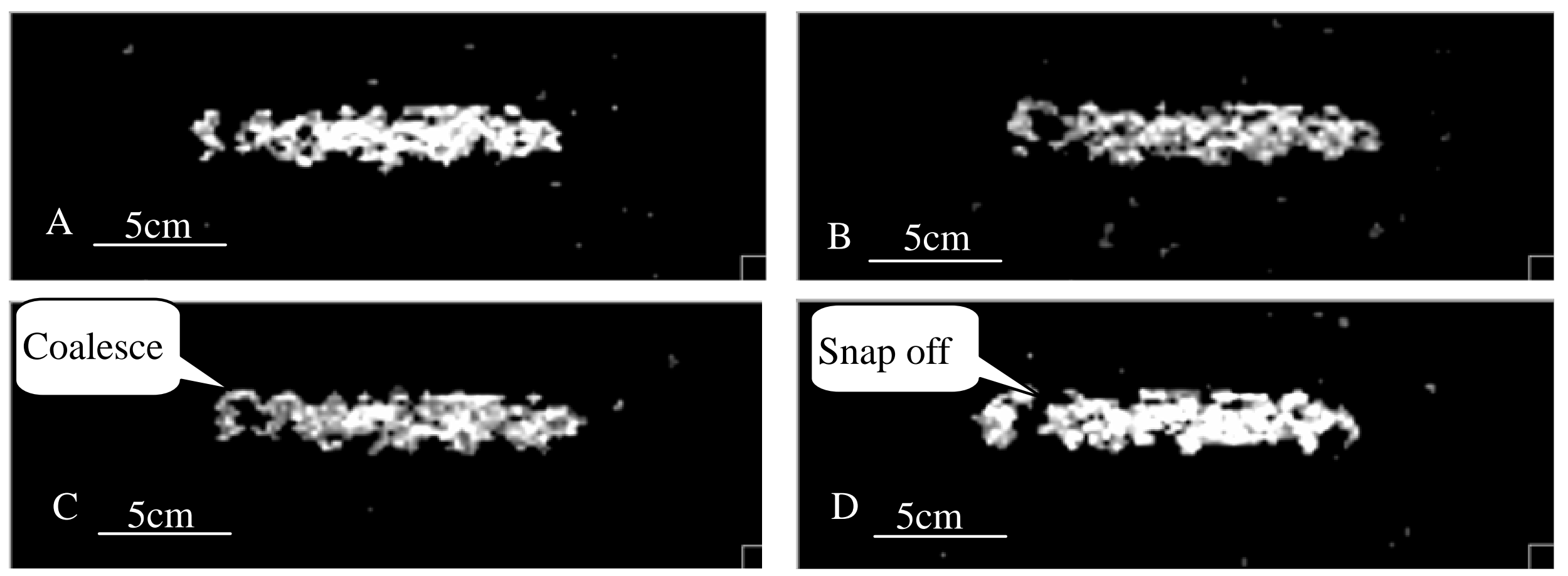


\section{Figure. 8}

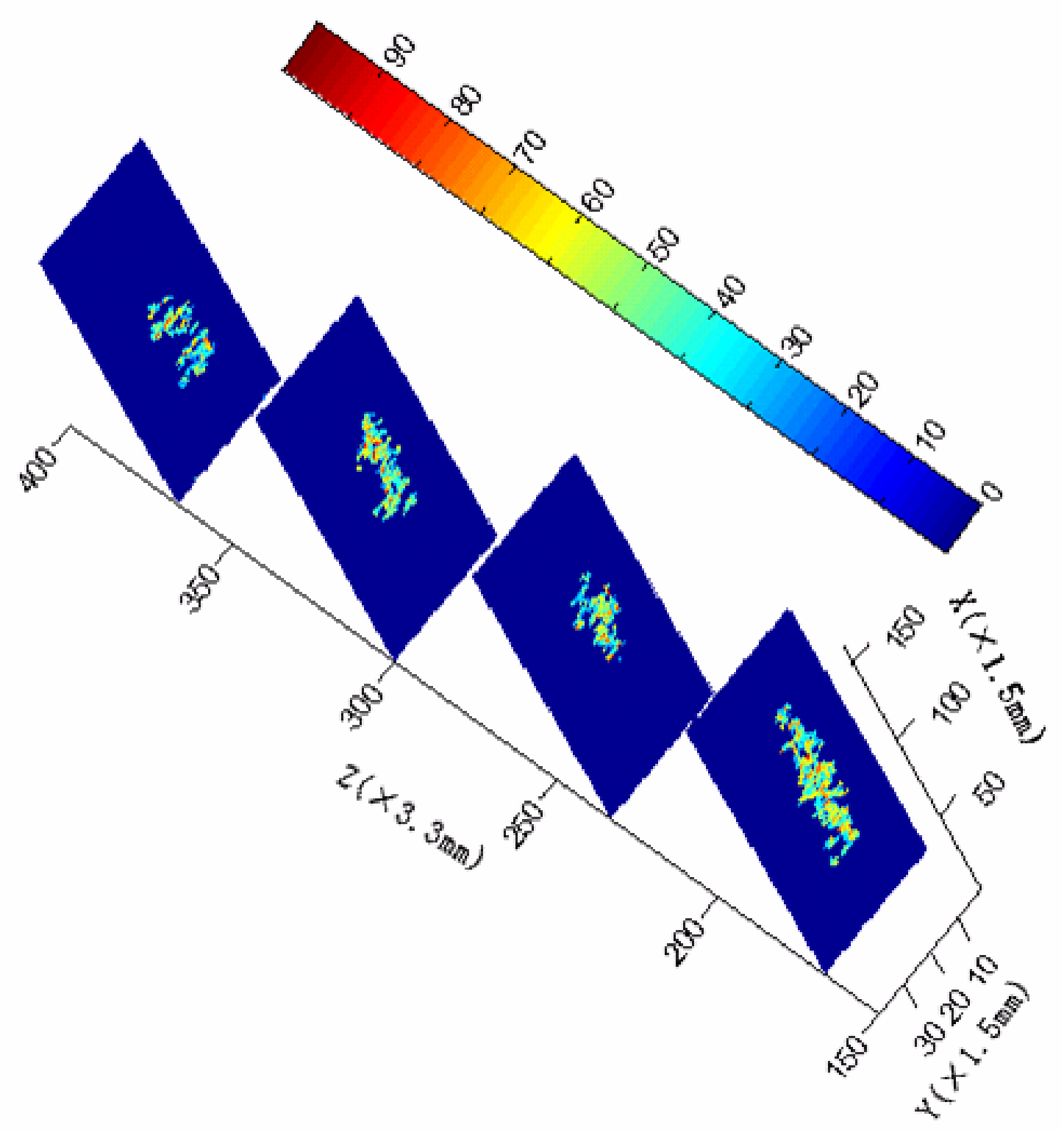




\section{Figure. 9}

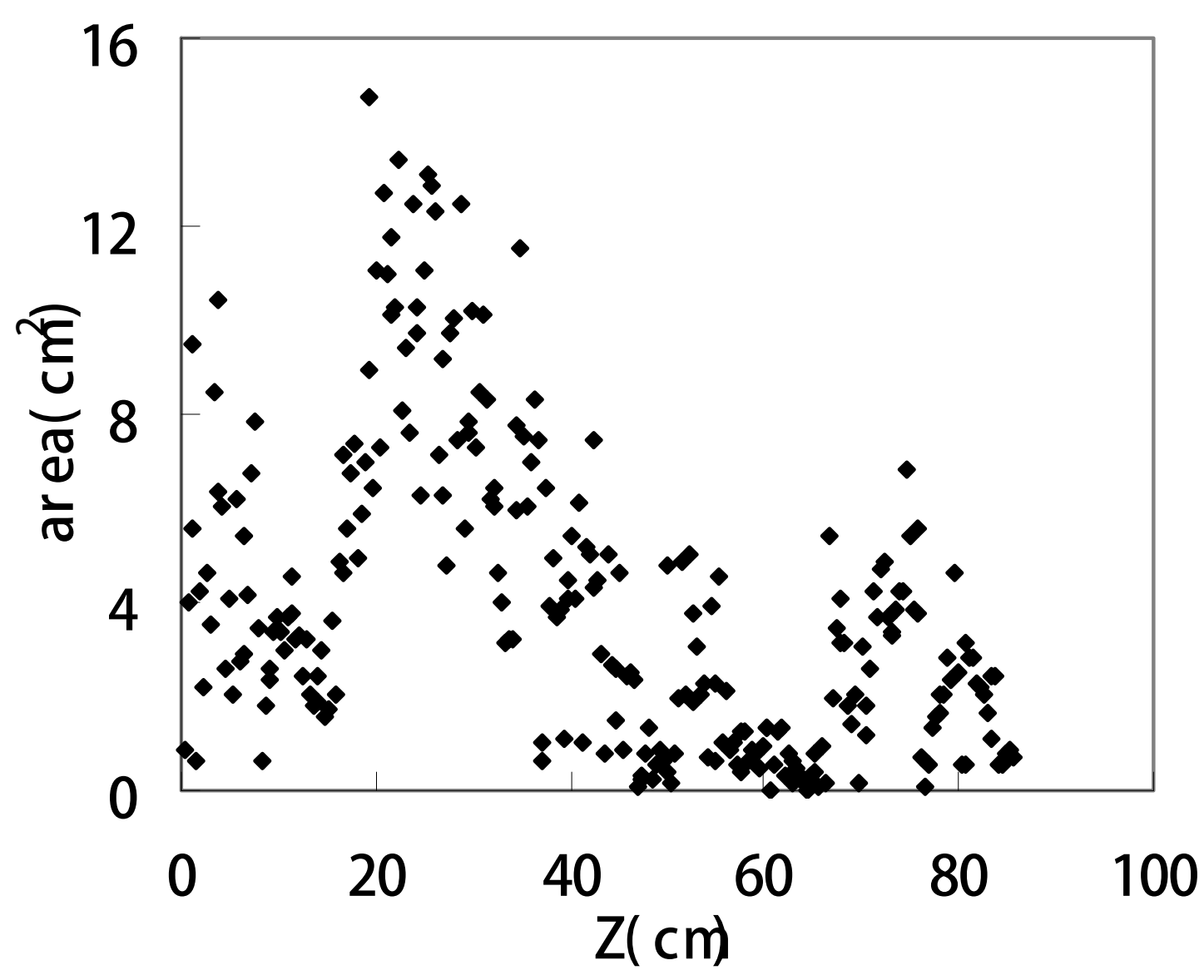




\section{Figure. 10}

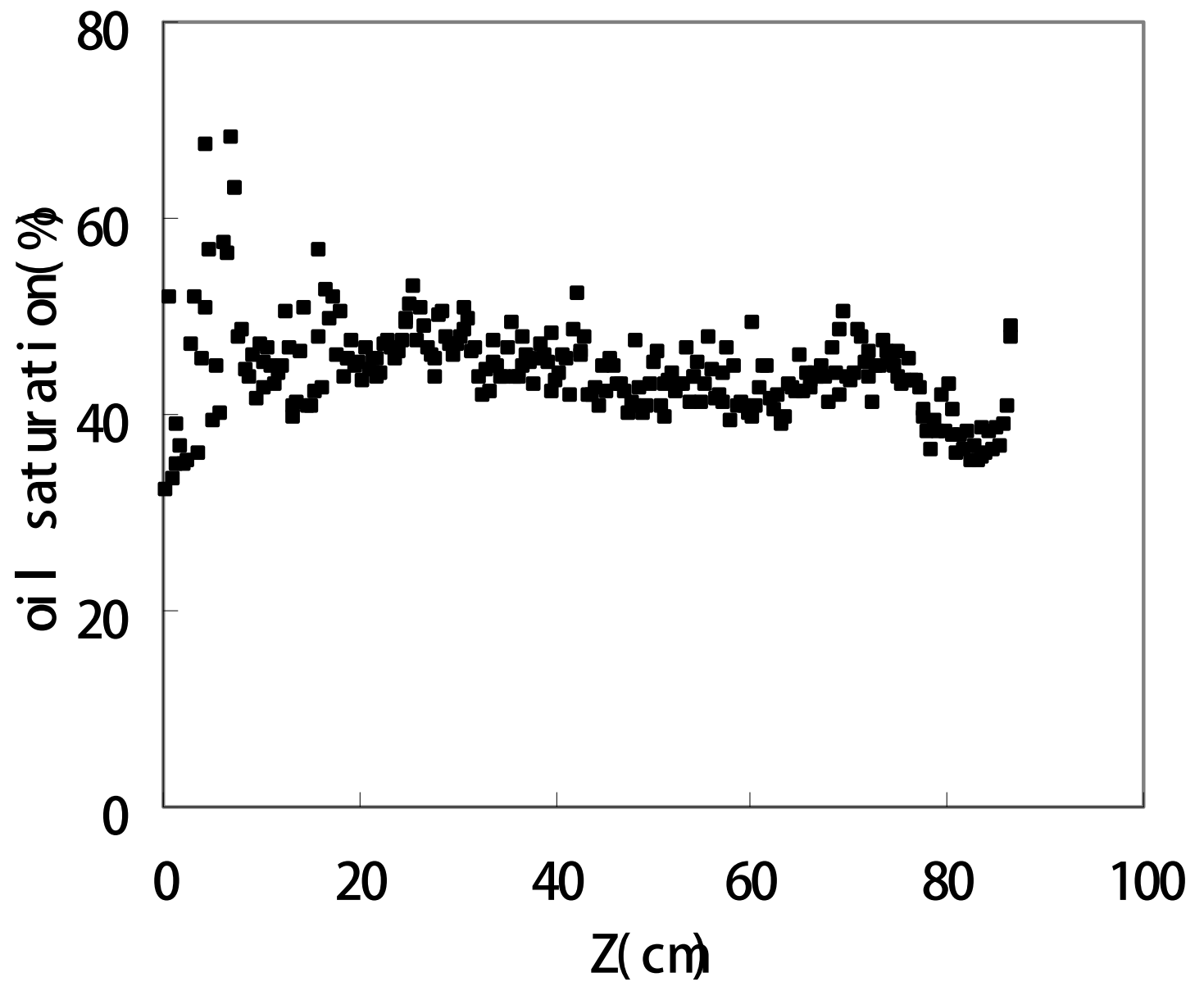




\section{Figure. 11}

\begin{tabular}{|c|c|c|c|}
\hline Item & Migration & Residual & Re-injection \\
\hline Slice & . & & \\
\hline Area( $\left.\mathrm{cm}^{2}\right)$ & 2.16 & 0.65 & 0.98 \\
\hline $\begin{array}{c}\text { Saturation } \\
(\%)\end{array}$ & 40.6 & 36.9 & 37.8 \\
\hline
\end{tabular}




\section{Figure. 12}

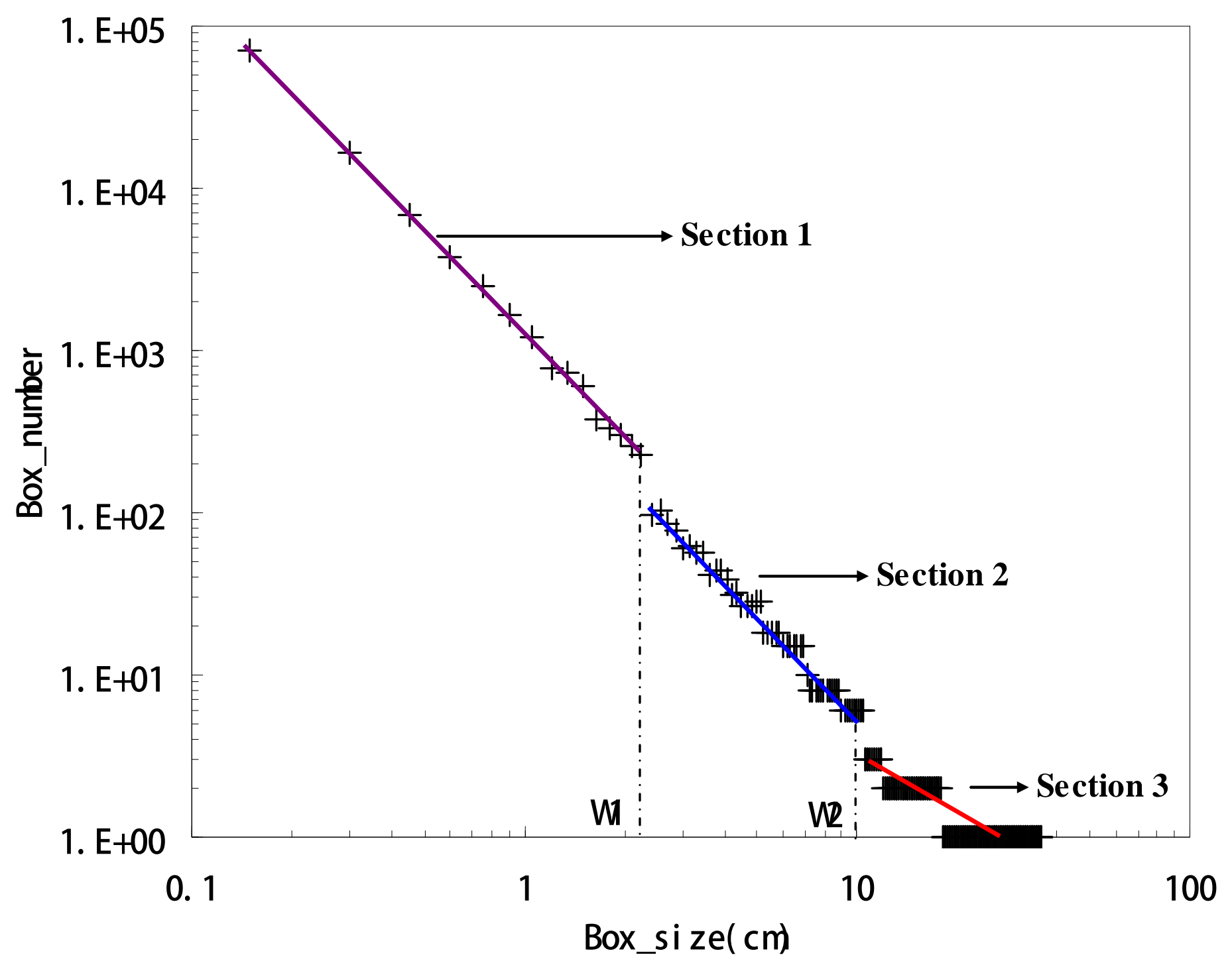

